ZHONGZHEN YANG, Ph.D. ${ }^{1,2}$

(Corresponding author)

E-mail: yangzz@dlmu.edu.cn

JIANNAN CHENG, Ph.D. ${ }^{3,4}$

E-mail: cheng_jian_nan@dlmu.edu.cn

${ }^{1}$ Faculty of Maritime and Transportation, Ningbo University, Ningbo, China

2 National Traffic Management Engineering \& Technology

Research Centre Ningbo University Sub-centre, Ningbo, China

${ }^{3}$ Transportation Management College,

Dalian Maritime University, Dalian, China

${ }^{4}$ Collaborative Innovation Center for Transport Studies

Dalian Maritime University
Science in Traffic and Transport Original Scientific Paper

Submitted: 1 Oct. 2016

Accepted: 3 July 2017

\title{
OPTIMIZATION OF TRIP-END NETWORKS AND RIDE PRICE FOR EXPRESS COACH SYSTEMS IN THE HIGH-SPEED RAIL ERA
}

\begin{abstract}
Express coach (EC) lost a considerable share of passengers after high-speed rail (HSR) was implemented. This paper proposes a door-to-door operation mode for the EC system and builds a model to design an EC trip-end network in the origin city with the aim of maximizing the EC's daily operating profit. A case study is undertaken, and the results show that the operating profit of the EC system first increases and then decreases with the growth of the trip-end routes. In the HSR era, door-to-door operation can effectively guarantee the market share and operating profits of the EC.
\end{abstract}

\section{KEY WORDS}

express coach; high-speed rail; door-to-door mode; trip-end network; ticket price; operating prof-it;

\section{INTRODUCTION}

\subsection{Background}

The rapid development of high-speed rail (HSR) has changed the supply system of intercity passenger mobility, and due to its advantages of high speed, safety and comfort, HSR has caused the market share of express coach (EC) in the same corridor to decrease significantly. Previous studies have shown that the loading rate of ECs was only $20 \%$ to $50 \%$ and business earnings decreased by $40 \%$ to $55 \%$ when HSR was implemented in the same corridor. Therefore, EC operators must address the challenges of HSR to maintain their market share and operating profits.

Chinese scholars have paid considerable attention to this issue and performed several studies on innovation for EC businesses. They have proposed six types of countermeasures: (1) improving the coach waiting conditions and services, computerizing the ticket system, and improving services; (2) forming large-scale trans-regional and cross-sector groups to realize scaled, specialized and intensive management; (3) increasing door-to-door services by providing access/ egress shuttle services and expanding the market to areas not covered by HSR; (4) constructing terminals to connect to other modes seamlessly and serve as the access or egress mode for HSR or flights; (5) focusing on segmented markets, such as tourism and travel, thus changing the profit model; and (6) controlling capacity at the macro-level and alleviating the control on on-board/off-board sites.

As shown by the above discussion, the existing literature has mainly addressed the problem at macro and strategic levels; however, the existing methods have been extremely broad and have lacked pertinence. Few of the studies have been quantitative with programing models. The unique attributes of $\mathrm{EC}$ have not been utilized effectively. Although many believe that EC must innovate their business and profit modes to the advantage of door-to-door services in the HSR era, no specific methods have been offered and no quantitative studies have been undertaken. For example, no literature has provided methods to optimize the EC transport network and to determine the EC fare system.

EC has high door-to-door accessibility (only outdone by that of private cars), whereas HSR is limited to the style of "station-to-station", and trip-end travel also influences the competitiveness of different modes. This paper innovates the operation mode of EC in the HSR era with a detailed scheme based on its door-todoor advantage, namely, by extending the routes with 
more boarding stops to the trip-ends and enabling passengers to board with lower access costs and to reach destinations without any transfers.

Increasing the number of stops on the extended routes means extending a single intercity EC route between two city centres to an EC network covering the two cities. By extending the route and the stops, the original axis-type EC route changes to a hub-spoke EC network to let the passengers travel from the origin trip-end to the destination trip-end without transfer. This EC network could expand the EC's spatial coverage and improve its accessibility without changing the depar-ture frequency. As a result, the original two trips of the intra-city trip-end trip and intercity main trip become a single door-to-door trip, saving transfer time and improving the efficiency of the EC.

Door-to-door EC service can increase this mode's operating efficiency and travel utility. This approach might be the most effective way to solve the operation dilemma of the EC in the HSR era. The design or optimization of the trip-end network for the EC is the key issue for the success of the new mode realizing doorto-door operations. This paper aims to create a programing model to optimize the trip-end network and fare system of the EC.

\subsection{Literature review}

There are limited existing studies addressing the design of networks for door-to-door EC operations. The door-to-door design involves the extension of EC routes into the origin or destination cities and determining the departure stops' locations. Thus, the design method of urban public transit networks (especially bus networks) may be applicable.

The optimization of urban bus networks has been widely studied. In the early period, the empirical method was used to design bus networks; in particular, the rationality of the network was determined by professional experience and subjective cognition. Due to the development of OR (Operational Research), some OR theories and methods were used, namely, optimization of bus networks with objective functions and some constraints. Lampkim [1] adopted comfort levels and travel times as the objectives to design a bus network and built a network optimization model. Mandl [2] argued that different objectives must be met in public transportation systems. According to Van Nes and Bovy [3], minimization of the total users and system costs is the most suitable and least complex objective when building a network optimization model. Some researchers have argued that transit network design is a multi-objective problem. Chakroborty and Dwivedi [4] combined indicators into a weighted summed value to determine the optimal transit network.
Follow-up studies have emphasized the use of a combination of planning methods and computing technology. Genetic algorithms, neural network techniques and other technologies have been used to solve bus network optimization problems. To find the optimal network, Ceder and Wilson [5] proposed an algorithm to study all possible routes between all origin/ destination (OD) pairs that complied with the service and operational constraints on trips, transfers, and waiting times. Baaj and Mahmassani [6] optimized bus networks with an artificial intelligence-based heuristic algorithm and achieved promising results. Additionally, Baaj and Mahmassani [7] adopted a heuristic approach that built one route at a time following a set of rules defined in a route generation heuristic algorithm (RGA) and considering the OD pair with the highest demand. Ceder and Israeli [8] used the shortest path algorithms to select candidate routes to optimize the transit network. Pattnaik et al. [9] designed a bus network to minimize the sum of the travelers' costs and operators' costs. They first generated network alternatives and then determined the best alternative using a genetic algorithm. Tom and Mohan [10] extended this algorithm by including new variables and coding schemes. Bielli et al. [11] designed a genetic algorithm to optimize a bus network with the objective of increasing the efficiency of the original network and reducing the number of bus vehicles used without increasing passengers' travel times. To configure a route network, Ngamchai and Lovel [12] developed a genetic algorithm and improved the optimization model simultaneously. Agrawal and Mathew [13] used the shortest path algorithms to select candidate transit routes. Considering both the user and the operator perspectives, Lee and Vuchic [14] proposed an iterative approach for sequentially determining routes for a transit network, enabling variability in transit demand to be considered. Dell'Olio et al. [15] proposed a bi-level mathematical programming model to micro-locate bus stops and optimize the frequencies considering that bus routes remain fixed, and the only decision variables were the micro-locations of the bus stops and the frequency determination. Fan and Machemehl $[16,17]$ compared different solution metaheuristics, including simulated annealing, taboo search, and genetic algorithms. They used a similar model formulation; in the first and second models, they used fixed demand, whereas they used changed demand in the third model.

Szeto and Wu [18] proposed an integrated solution method that combines a genetic algorithm and neighborhood search heuristic to tackle bus network design problem, including route design and frequency setting. Numerical study shows that the number of transfers and the total travel time of the users were reduced by $20.9 \%$ and $20.9 \%$, respectively. Afandizadeh et al. [19] used genetic algorithms to solve the bus network 
design problem, which included the frequency setting and assignment procedure and network evaluation. The authors also uniquely considered passenger as-signment using a travel time utility when at least two transit options existed. Szeto and Jiang [20] proposed a bi-level model that considered in-vehicle congestion to design transit routes and their frequencies. The upper-level problem aims to minimize the total number of passenger transfers, and strict capacity constraints are added to the lower-level problem. Arbex and da Cunha [21] proposed an alternating objective genetic algorithm to efficiently solve the multi-objective transit network design problem, aiming to minimize both passengers' and operators' costs, and the results indicated that the algorithm led to improved solutions. Zhang et al. [22] presented an improved matrix multiplication method to solve the all-pairs-shortest-path problem in the pulse coupled neural network.

The existing studies on the optimization and design of bus networks have provided some promising methods for designing trip-end networks for intercity coach systems. However, some gaps still exist between urban bus transit and EC systems. For example, the urban bus system is a part of the public welfare, and its primary purpose is not to gain profit but to offer citizen mobility services and encourage travellers not to use private cars as a trip mode. In contrast, both demand from passengers and operating profits must be considered when designing the trip-end network of the EC system. The reason for considering passengers' demand is also to maximize the operation revenues and operating profits, indicating that the design of the tripend networks of EC systems still has unique requirements. We must overcome problems based on the existing studies while considering their unique attributes more seriously. The goals are to include the ticket price in the trip-end network design and to obtain an overall coach operating network that will allow the EC to earn suitable profits and to be able to compete with HSR.

Based on the above background, in this paper, we consider the optimization of the trip-end network of ECs in the origin city to allow ECs to offer a door-todoor service by considering the profits of both the operator and the passengers. The aims are to establish the trip-end network configuration (including routes and corresponding stops) and determine the ticket prices. To test whether the door-to-door mode and network design method could be used in the real world, we have performed a case study with data from Dalian to demonstrate the feasibility and effectiveness of the method. The contributions of this paper are as follows: (1) proposing a new business model for ECs to maintain their market share and profitability in the HSR era; (2) simultaneously optimizing the routes, stop locations and corresponding ticket prices of ECs with a bi-level programming model, which cannot only allow the operator to obtain profits but also increase ECs' service quality and decrease travellers' costs; and (3) partitioning the origin city into radiated fan-shaped areas to provide a method for determining the number of the end-trip routes and their running directions.

\section{PROBLEM DESCRIPTION}

The optimization of the trip-end network of an EC system is a complex decision-making problem, in which the operators' profits and passengers' demand must be balanced. In particular, the interaction between profit and demand should be fully considered. The EC's operator always wants to maximize profits, whereas travellers from the demand side typically pursue the maximum utility or minimum total cost. The interaction between the behaviours of the operator and the passengers can be regarded as a bi-level programming problem with a master-slave hierarchical structure. Therefore, we can optimize the trip-end network of the EC system using a bi-level programming model. For optimization, the upper model provides the network structure, and the lower model simulates the path choice behaviours of the passengers using user equilibrium theory to obtain the market scale, operators' revenues and business profit. The designer can determine whether the network provided in the upper model is reasonable based on the profit and passenger travel utility and then feeds the results back to the upper model.

Figure 1 shows the EC trip-end network for which two EC routes and the path selection of the passengers at origin G1 are given in detail. After the interaction between the upper model and the lower model,

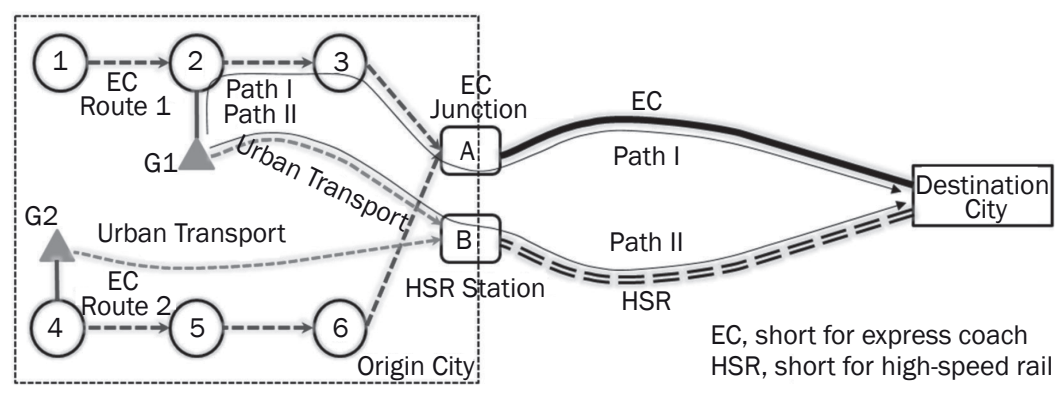

Figure 1 - The EC network in an interaction between the upper and lower models 


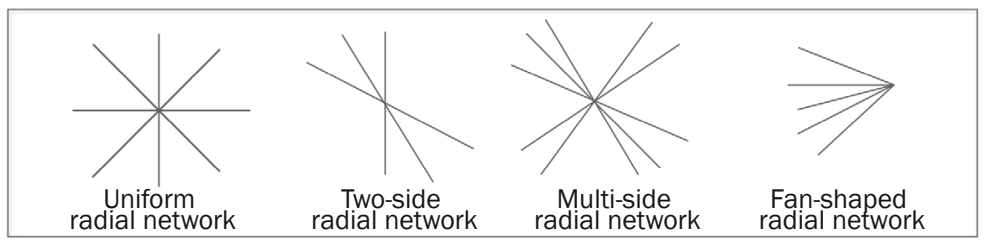

Figure 2 - Shapes of radial trip-end networks

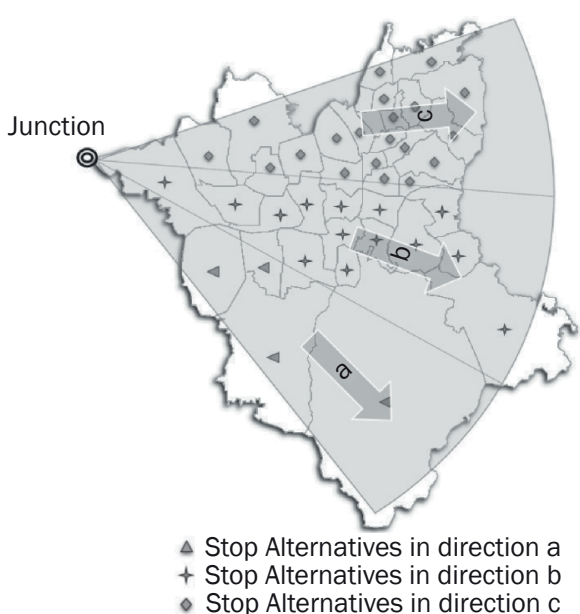

Figure 3 - Running directions and stop locations of the EC's trip-end network

the trip-end network of the EC system is generated by a heuristic algorithm in the upper model, whereas the chosen paths are obtained by assigning the trips with the user equilibrium principle in the lower model. Passengers from G1 will choose travel paths based on the traffic impedance. Because traffic impedance can change with link flows, some passengers will travel along path I, whereas other passengers will travel along path II.

For convenience, we assumed that there is only one stop in the destination city; this assumption does not affect the validity of the model. Because the outputs of the upper and lower models interact, the calculations must be repeated iteratively. The optimal EC trip-end network and fare system can be obtained when the interactive relationship between the upper and lower models reaches the equilibrium.

The tasks and difficulty of the upper model are to determine the routes and stops in the origin city with the objective of maximizing the operating profits and building a trip-end network that covers as many travellers as possible to allow them to board the EC conveniently. In the old operation mode, there was only one coach stop in the trip-end city, and passengers had to reach the EC by urban transport from their origins. Now, the passengers can enjoy door-to-door service via the trip-end network of the EC system. The trip-end network of the EC system can be divided into three types: radial, grid and ring [23]. Among them, the radial type is the most prominent due to its all-direction coverage and greater accessibility. We use the radial network to set the alternatives of the trip-end network. The radial network can be further subdivided into 4 types, uniform, two-sided, multi-sided, and fanshaped, as shown in Figure 2 [24].

Since the trip-end network generally terminates at the EC junctions in the origin or destination cities and the junctions are mainly located at the city's outer edges and radiate from the city in a single direction, the trip-end network should be a fan-shaped radiation, particularly with the running direction of the feasible routes arranged in a radiated, fan-shaped area. Thus, to determine the feasible trip-end routes in the upper model, we can cover the entire city with fan-shaped sectors by considering the junction as the circle centre and placing one route in each sector to form the network alternatives. For example, in Figure 3, Sectors a, $\mathrm{b}$ and $\mathrm{c}$ are the three sectors covering the entire area, and the centroids of traffic zones within the sectors are the sets of stop alternatives on different route alternatives.

\section{MATHEMATICAL MODEL}

The objective of the upper model is to maximize the operator's daily business profit, and that of the lower model is to minimize the passengers' generalized travel costs. Here, some assumptions are made for the modelling as follows: (1) Only ECs and HSR are available for intercity passenger transport, and the ticket price and operating frequency of HSR are provided; (2) The central point of an administrative unit is set as the EC's departure stop; (3) The ticket price from the passenger's origin (home) to EC's departure stop or HSR station is ignored.

\subsection{Variables and parameters}

- EC's departure stop alternatives in the origin city

$t_{1}$ - EC junction (toll gate)

$t_{2}$ - HSR station

D - Destination city

$B \quad-\left\{O, t_{1}, d\right\}$

H $\quad-\left\{0, t_{2}, d\right\}$

$R \quad$ - Set of EC and HSR routes

$\mathrm{K}$ - Set of feasible trip-end routes of the $\mathrm{EC}$ in the origin city

$n \quad-$ Number of feasible routes in the EC trip-end network 
m - Number of stops on one feasible EC route

$S_{k} \quad$ - Set of departure stops on the $k$-th feasible EC route

$l_{i j}^{k} \quad$ - Distance between departure stop $i$ and stop $j$ on the $k$-th feasible EC route

$I_{i j}^{k} \quad$ - Distance between departure stop $i$ and stop $j$ on the $k$-th feasible EC route

$I^{k} \quad$ - Linear distance of the $k$-th feasible EC route

$I_{\min }, k_{\max }-$ Permitted minimum/maximum distances between adjacent departure stops, respectively

$Z_{\max } \quad$ - Permitted maximum non-linear coefficient of a feasible EC route

$D_{\max } \quad$ - Permitted maximum departure stops of a feasible EC route

C - Daily cost per EC

W - Capacity per EC

A - C's actual loading rate

F $\quad$ - Permitted daily minimum dispatched ECs for one feasible EC route

$Q^{k} \quad$ - Daily dispatched ECs on the $k$-th feasible EC route

$X^{k} \quad$ - Daily operation duration of the $k$-th feasible EC route

$q_{i}^{k} \quad$ - Total passenger flows in the administrative unit where departure stop $i$ on the $k$-th feasible EC route is located

$q_{b i}^{k} \quad$ - Total passenger flows carried by EC from the administrative unit where departure stop $i$ on the $k$-th feasible EC route is located

$q_{h i}^{k} \quad-$ Total passenger flows carried by HSR from the administrative unit where stop $i$ on the $k$-th feasible EC route is located

$p_{b i} \quad-$ EC ticket price from EC junction (toll gate) to destination city, which is defined as the EC basic ticket price

$p_{b i 0} \quad-$ EC initial ticket price from departure stop $i$ to the destination city

$p_{h i} \quad$ - HSR ticket price from departure stop $i$ to the destination city

$p_{h 0} \quad-$ HSR ticket price from HSR station to the destination city, which is defined as the HSR basic ticket price

$q_{b i j} \quad$ - Passenger flow on EC link $(i, j)$

$q_{h i j} \quad$ - Passenger flow on HSR link $(i, j)$

$L_{i j} \quad$ - Capacity on EC link $(i, j)$

$U_{i j} \quad$ - Capacity on HSR link $(i, j)$

$Q_{o d} \quad$ - Total passenger flow from origin o to destination city $d$

$f_{r}^{\text {od }} \quad$ - Passenger flow on the $k$-th path from origin $o$ to destination city $d$

$z_{b i j}(w)$ - Impedance of EC (including access urban road) links

$z_{\text {hij }}(w)$ - Impedance of HSR links

$\alpha, \beta \quad$ - Updated coefficients of ticket price
VOT - Value of time, calculated using real data from the study area

$\delta_{i j, r}^{o d}= \begin{cases}1 & \text { if link }(i, j) \text { is on the } k \text { - th path from origin o } \\ & \text { to destination city } d \\ 0 \quad & \text { otherwise }\end{cases}$

1 if alternative departure stop $i$ is chosen as

$x_{i}^{k}= \begin{cases} & \text { the stop on } k \text { - th feasible route } \\ 0 & \text { otherwise }\end{cases}$

$y_{i j}^{k}=\left\{\begin{array}{l}1 \\ 0\end{array}\right.$ if link $(i, j)$ is on the $k$ - th feasible EC route

$\sigma_{k}=\left\{\begin{array}{l}1 \text { if the } k \text { - th feasible route is chosen as } \\ \text { the route of EC tripend network } \\ 0 \quad \text { otherwise }\end{array}\right.$

\subsection{Upper model}

Feasible trip-end routes of the EC system

First, the running directions of the feasible EC routes are determined. Radial sectors with the expressway junction are used at the origin city as the circle centre to cover the entire city and consider each sector to represent a running direction, with one tripend route being set correspondingly. As a result, the set of feasible trip-end routes is obtained. The routes must satisfy the following requirements:

$\frac{\sum_{i \in 0} \sum_{j \in O} l_{i j}^{k} y_{i j}^{k}+\sum_{i \in O} l_{i t_{1}}^{k} y_{i t 1}^{k}}{l^{k}} \leqslant Z_{\max }, \quad \forall k \in K$

$\frac{\sum_{i \in O} x_{i}^{k} q_{i}^{k}\left(1+\sum_{j>i} x_{j}^{k}\right)}{\sum_{i \in O} x_{i}^{k} q_{i}^{k}} \leqslant D_{\max }, \quad \forall k \in K$

$I_{\min } \leq I_{i j}^{k} \leq I_{\max }, \quad \forall k \in K$

$\sum_{i \in O} x_{i}^{k}=m, \quad \forall k \in K$

$x_{i}^{k} \in\{0,1\}, \quad \forall i \in 0, \quad \forall k \in K$

$y_{i j}^{k} \in\{0,1\}, \quad \forall i \in 0, \forall j \in 0$, or $j=t_{1}, \forall k \in K$

Equation 1 is the route curve constraint; Equation 2 is the coach stopping times constraint, indicating that the weighted average stopping times of the passengers should be less than a given number. Because stopping might make passengers uncomfortable and waste time, excessive stopping can reduce the service level. Equation 3 is the distance constraint between two stops, Equation 4 is the stop number constraint, and Equations 5 and 6 are the variable constraints.

Trip-end network of the EC system

Different trip-end networks can be obtained by combining different route alternatives after determining the set of feasible routes. Then, the trip-end 
network in the origin city can be optimized with the aim of maximizing the operator's daily business profit. The model is as follows:

Max: $\sum_{k \in K} \sum_{i \in S_{k}} p_{b i}^{k} q_{b i}^{k} \sigma_{k}-\sum_{k \in K} C Q^{k} \sigma_{k}$

s.t. $\quad \sum_{k \in K} \sigma_{k}=n$

$Q^{k}=\frac{\sum_{i \in s_{k}} q_{b i}^{k}}{W \cdot A}, \quad \forall k \in K$

$\sigma_{k} \in\{0,1\}, \quad \forall k \in K$

Equation 7 is the objective function, which maximizes the daily operating profit, where the first part is operator's daily business revenue and the second part is the daily operating cost. Equation 8 is the route number constraint, Equation 9 is the coach dispatching frequency, and Equation 10 is the variable constraint.

\subsection{Lower model}

There is feedback between the passenger volume and the ticket price of an EC route. In other words, increasing the ticket price will reduce passenger utility and decrease demand; conversely, reducing the ticket price may increase demand. Therefore, the upper model is designed to describe the feasible schemes of the price and network and further measure the EC's performance corresponding to each scheme. Accordingly, the lower model is used to find the paths for the passengers. The equilibrium scheme is where changes in the price and network do not improve the EC's profit. Then, the ticket price and network in this scheme are taken as the optimized values.

The path choice model is as follows:

$$
\begin{aligned}
\text { Min: } & \sum_{i \in B} \sum_{j \in B} \int_{0}^{q_{b i j}} z_{b i j}(w) d w+ \\
& \sum_{i \in H} \sum_{j \in H} \int_{0}^{q h i j} z_{h i j}(w) d w
\end{aligned}
$$

s.t. $\quad \sum_{r \in R} f_{r}^{o d}=Q_{o d}, \quad \forall o \in O$

$f_{r}^{o d} \geq 0, \quad \forall 0 \in 0$

$q_{b i j} \leq L_{i j}, \quad \forall i, \quad j \in B$

$q_{h i j} \leq U_{i j}, \quad \forall i, \quad j \in H$

$q_{b i j}+q_{h i j}=\sum_{o \in O} \sum_{r} f_{r}^{o d} \delta_{i j, r}^{o d}$

$z_{i j}=p_{i}+V O T \cdot t_{i j}$

$p_{b i}=\left\{\begin{array}{cl}p_{b i 0}, & q_{b i j} \leq \alpha \cdot L_{i j} \\ p_{b i 0}\left(\frac{q_{b i j}}{\alpha \cdot L_{i j}}\right)^{\beta} & q_{b i j}>\alpha \cdot L_{i j} \\ p_{b i 0}=p_{b 0}\left(1+\frac{l_{i t 1}}{I_{t 1 d}}\right)\end{array}\right.$

$p_{h i}=p_{h 0}$

$t=t_{r}+t_{w}+t_{o}$
Equation 11 is the objective function. Equation 12 states that the sum of passenger flows on all paths between an od pair equals the od passenger flow. Equation 13 is the variable constraint, which states that passenger flows on all paths between an od pair are non-negative. Equations 14 and 15 state that the passenger flows on each link should be less than the service capacity of EC and HSR. Equation 16 indicates that the link flow is the accumulation of the path flows. Equation 17 shows that the traffic impedance of link $(i, j)$ is determined by the ticket price and total time spent by a passenger when a transport mode is chosen.

Equation 18 represents the EC ticket price from departure stop $i$ to the destination city. It is influenced by two factors: distance and passenger flow. (1) The influence of distance is as follows: the EC initial ticket price varies for different departure stops; it is determined by the EC basic ticket price and the distance between the departure stop and the EC junction, which can be calculated by $p_{b i 0}=p_{b o}\left(1+\frac{l_{i t_{1}}}{l_{t 1 d}}\right)$, where $l_{i t_{1}}$ denotes the distance between departure stop $i$ and the EC junction; $I_{t_{1} d}$ denotes the distance between the EC junction and the destination city, and $p_{b 0}$ represents the EC basic ticket price. (2) The influence of passenger flow is as follows: based on the idea of revenue management, when the passenger flows on link $(i, j)$ are equal to or less than $\alpha$ of the EC capacity, namely, not reaching the presupposed congestion degree, the ticket price of departure stop $i$ is its EC initial ticket price $p_{b i 0}$; otherwise, the ticket price of departure stop $i$ is calculated by $p_{b i}=p_{b i 0}\left(\frac{q_{b i j}}{\alpha \cdot L_{i j}}\right)^{\beta}$.

Equation 19 shows that the HSR ticket price from departure stop $i$ to the destination city is a constant, which is the HSR basic ticket price. Equation 20 represents the total time spent by a passenger travelling from departure stop $i$ to the destination city, including time $t_{r}$ required by the passenger to get to the "station" (station means departure stop $i$ when choosing EC and the HSR station when choosing HSR), the waiting time of feasible route $k, t_{w}=X^{k} / Q^{k}$ and the vehicle travelling time $t_{0}$.

\section{SOLUTION METHODS}

To conduct a practical analysis, the dual-population genetic algorithm is designed to solve the upper model, and the Frank-Wolfe algorithm is used to solve the lower model. The calculation processes are as follows.

\subsection{Upper model - dual-population genetic algorithm}

The genetic algorithm is an efficient random search algorithm to solve the optimization problem, with a strong global searching capability and high fault 
tolerance. However, the standard genetic algorithm (SGA) can easily result in premature convergence in cases of small population sizes. In contrast, the dual-population genetic algorithm (DPGA) reduces the possibility of premature convergence [25]. The DPGA generates 2 initial populations of chromosomes with the same coding forms and population sizes, and each population proceeds to "crossover, mutation" separately, whereas a "Mass Selection" method is used in the selection to maintain the diversity of each population. Thus, we use the DPGA to solve the above model, and the calculation process is as follows.

\section{Step 1: Population initialization}

\section{1) Parameter setting}

Two groups of populations are generated, each of which has 100 individuals ( $=100$ ). The numbers of feasible trip-end routes are set at 2, 3, 4 and 5, and each feasible trip-end route has 5 stops; thus, the lengths of the individual chromosomes are 10, 15, 20 and 25, respectively. The calculation iteration is set at 500; the mutation probability is set at 0.05 .

2) Encoding

The "real-number encoding" method is adopted. The chromosome includes $n$ parts, where $n$ is the number of feasible routes in the trip-end network. Figure 4 shows an example of $n=3$, where Parts 1 , 2 and 3 represent feasible routes and the number of genes is determined by the stop numbers of the feasible routes (here, it is 5 ). Thus, the total number of genes when $n=3$ is 15 . The setting method of each part is similar; taking Part 1 as an example, the fourth and fifth genes represent the junctions in the origin and destination cities, respectively. Genes 1-3 represent stops inside the origin city, among which gene 1 represents the first stop and genes 2-3 represent randomly generated stops. In conclusion, the encoding of Figure 4 indicates that there are 3 feasible routes: Route 1 is 20-27-17$37-39$, Route 2 is 14-3-11-37-39, and Route 3 is 8-12-6-37-39.

\begin{tabular}{|l|l|l|l|l|l|l|l|l|l|l|l|l|l|l|}
\hline 20 & 27 & 17 & 37 & 39 & 14 & 3 & 11 & 37 & 39 & 8 & 12 & 6 & 37 & 39 \\
Part 1
\end{tabular}

Figure 4 - Encoding method of each feasible route

\section{Step 2: Fitness evaluation}

We use the value of the objective function in the upper model (Equation 7) as the fitness value, indicating the daily operation profit of the EC.

Step 3: Genetic algorithm operations

1) Selection

"Mass selection" is used to (1) place the two groups of chromosomes in one pool, and (2) generate two new groups of chromosomes by selecting chromosomes from the pool; during the selection, one chromosome cannot be placed in one group twice. Moreover, we adopt the elitism strategy, namely, placing the outstanding chromosomes into new groups, to avoid losing good genes and deteriorating the chromosome.

2) Crossover Crossover is undertaken as follows:

a) We pair the 100 chromosomes to obtain 50 pairs $(i=\{1,2, \ldots, 50\})$ of chromosomes, and the paired chromosomes are represented as $r_{1}$ and $r_{2}$.

b) When $i=1$, we take the first gene $r_{11}$ in Part 1 of $r_{1}$ as the first gene $r_{11}$ in Part 1 of the child chromosome $r_{1}^{\prime}$;

c) At the same time, we search the location of gene $r_{11}$ in Part 1 of $r_{2}$. If it is found, then we mark it as $r_{2 k}$ and then determine whether the genes in positions $r_{12}$ and $r_{2(k+1)}$ are chosen as the child genes; if none are chosen, we use the gene with the shortest stop spacing as the second gene in Part 1 of child chromosome $r_{1}{ }^{\prime}$; if one is chosen as the gene of child chromosome $r_{1}{ }^{\prime}$, we designate the other one to be the second gene in Part 1 of child chromosome $r_{1}^{\prime}$; if both are chosen as the genes of child chromosome $r_{1}{ }^{\prime}$, we move to the next gene positions in $r_{1}$ and $r_{2}$ and determine whether the two new genes are the child genes or not, until the new child gene is chosen.

d) After operation of Parts 1 and 2 of $r_{1}$ and $r_{2}$, we move forward to Parts 2 and 3 with the same operation.

e) We let $i=i+1$ and repeat steps b) and c) until $i=50$; then, we stop this round of the crossover operation.

3) Mutation

"Real value mutation" is conducted with a mutation rate of $P_{m}=0.05$ for a selected individual. One gene is mutated in Parts 1, 2 and 3 of the individual. The mutated individual should satisfy the constraints of the upper model.

Step 4: Optimal solution

We repeat Steps 2 and 3 with the new population until the iteration equals maximum, and the individual with the max value of fitness value is output as the optimal solution.

\subsection{Lower model - Frank-Wolfe}

The Frank-Wolfe algorithm is used to solve the lower model. The calculation is as follows:

Step 1: Initialization: Set $Z_{i j}{ }^{0}=Z_{i j}(0), \forall \operatorname{Link}(i, j)$, and perform an all-or-nothing assignment to obtain a set of feasible flows $\left\{x_{i j}^{1}\right\}$ and set $n=1$;

Step 2: Link-impedance update: $\operatorname{set} Z_{i j}^{n}=Z_{i j}\left(x_{i j}^{n}\right), \forall \operatorname{Link}(i, j)$. Step 3: Direction finding: perform an all-or-nothing assignment again with $Z_{i j}^{\mathrm{n}}=Z_{i j}\left(x_{i j}^{\mathrm{n}}\right), \forall \operatorname{arc}(i, j)$ to additional link flows $\left\{y_{i j}^{n}\right\}$; 
Step 4: Move-size determination: solve the following equal to obtain $\lambda$ :

$$
\sum_{i j}\left(y_{i j}^{n}-x_{i j}^{n}\right) \cdot Z_{i j}\left[x_{i j}^{n}+\lambda\left(x_{i j}^{n}-x_{i j}^{n}\right)\right]=0
$$

Step 5: Flow update: $x_{i j}^{n+1}=x_{i j}^{n}+\lambda\left(y_{i j}^{n}-x_{i j}^{n}\right)$.

Step 6: Convergence judgment. If $\frac{\sum_{i j}\left(x_{i j}^{n+1}-x_{i j}^{n}\right)^{2}}{\sum_{i j} x_{i j}^{n}<\varepsilon}$ ( $\varepsilon$ : a given threshold), stop the calculation and output $x_{i j}^{n+1}$. Otherwise, set $n=n+1$ and go to Step 2 .

\section{CASE STUDY}

We performed a case study with the actual passenger data from Dalian to Shenyang to demonstrate the use of the new mode and to explore the optimal network of the trip-end routes.

\subsection{Data}

\section{Study area}

The central Dalian area (approximately $300 \mathrm{~km}^{2}$, consisting of the districts of Ganjingzi, a high-tech zone, Shahekou, Xigang, Zhongshan, Lvshunkou) was considered as the study object. The area is divided into 51 administrative units. We numbered the units with the population order shown in Figure 5a. According to the populations of the units and geographical locations, the less important units were eliminated, and the remaining 36 units were used as the traffic zones and were re-numbered from 1 to 36 . Moreover, the expressway junction and Dalian HSR station were numbered 37 and 38, and the destination city (Shenyang) was numbered 39 , as shown in Figure $5 b$. Then, the set of trip-end routes was determined by reference to the radial sectors with the junction as the circle centre. Here, the origin city was covered by 3 radial sectors; thus, 3 route alternatives were obtained accordingly (Figure 5b), and three stops were supposed to be set along each route alternative.

\section{Basic data}

The express toll of a single coach trip from Dalian to Shenyang is 550 yuan, and the fuel cost is 500 yuan ( $5 \mathrm{~L} /$ yuan $\times 25 \mathrm{~L} / 100 \mathrm{~km} \times 400 \mathrm{~km}$ ). The life span of a coach is 8 years, and the purchasing price is 800,000 yuan; thus, the daily depreciation value of a coach is approximately 300 yuan, and the depreciation value of a coach during a single trip is 150 yuan. The staff cost of a coach is 400 yuan/day, and the staff cost of a single trip is 200 yuan/day. Therefore, the operating cost of a single coach trip from Dalian to Shenyang is $C=1,400$ yuan.

We assumed that the average EC speed in the study area is $30 \mathrm{~km} / \mathrm{h}$, the average bus speed in the study area is $15 \mathrm{~km} / \mathrm{h}$, the capacity per EC is $W=50$, the EC actual loading rate is $A=70 \%, t_{b r}=10 \mathrm{~min}, t_{b w}=15 \mathrm{~min}$, $t_{h r}$ is the ratio of the distance between the origin and HSR station and the average bus speed, $t_{h w}=45 \mathrm{~min}$ (the average of the survey data), the capacity of EC link $(i, j)$ is $L_{i j}=1,000$, and the capacity of HSR link $(i, j)$ is $U_{i j}=10,000$.

Based on the practical situation, the ticket price of HSR is set to 180 yuan, the EC basic ticket price is set to 85 yuan, and the EC initial ticket price of each departure stop is calculated by Equation 19, which is in the range of 85 to 89 yuan. Considering the existing EC ticket price and its attractiveness to passengers, the EC maximum ticket price of departure stop i is set to 110 yuan, that is to say, when the EC ticket price is greater than 110 yuan, the passenger will choose HSR rather than $\mathrm{EC}$.

The EC ticket price of departure stop $i$ can be calculated using Equation 18 when the passenger flows of link $(i, j)$ are changed. There is no standard for $\alpha$; we assumed that when the passenger flows exceed $60 \%$ of the capacity on EC link $(i, j)$, the EC ticket price of departure stop $i$ should be increased, namely, $\alpha=0.6$. When the passenger flow on EC link $(i, j)$ reaches saturation, the EC ticket price of departure stop $i$ is $p_{b i}=110$ yuan, and the data of the ticket price and passenger flows under saturation are put into Equation 18, which produces $\beta=0.41$.

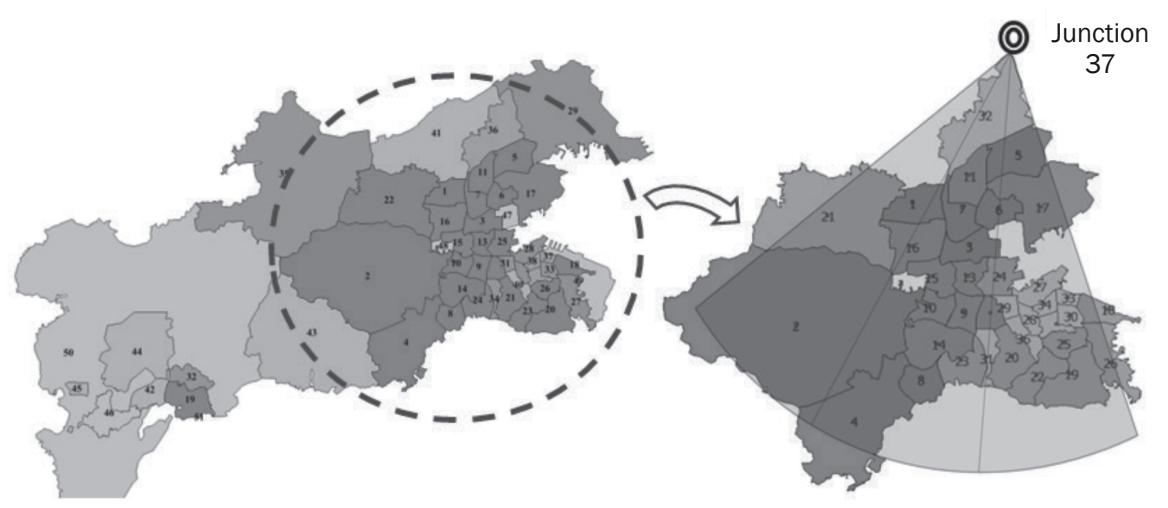

a)

b)

Figure 5 - a) Case study area; b) Slim case study area 
Table 1 - Passenger volume in the traffic zones (April 2015: person/day)

\begin{tabular}{||c|c||c|c||c|c||c|c||}
\hline $\begin{array}{c}\text { Traffic } \\
\text { zone }\end{array}$ & $\begin{array}{c}\text { Passenger } \\
\text { volume }\end{array}$ & $\begin{array}{c}\text { Traffic } \\
\text { zone }\end{array}$ & $\begin{array}{c}\text { Passenger } \\
\text { volume }\end{array}$ & $\begin{array}{c}\text { Traffic } \\
\text { zone }\end{array}$ & $\begin{array}{c}\text { Passenger } \\
\text { volume }\end{array}$ & $\begin{array}{c}\text { Traffic } \\
\text { zone }\end{array}$ & $\begin{array}{c}\text { Passenger } \\
\text { volume }\end{array}$ \\
\hline \hline 1 & 495 & 10 & 324 & 19 & 263 & 28 & 203 \\
\hline 2 & 462 & 11 & 322 & 20 & 261 & 29 & 150 \\
\hline 3 & 456 & 12 & 317 & 21 & 240 & 30 & 146 \\
\hline 4 & 442 & 13 & 300 & 22 & 237 & 31 & 137 \\
\hline 5 & 377 & 14 & 293 & 23 & 237 & 32 & 136 \\
\hline 6 & 373 & 15 & 279 & 24 & 232 & 33 & 135 \\
\hline 7 & 336 & 16 & 278 & 25 & 217 & 34 & 134 \\
\hline 8 & 334 & 17 & 276 & 26 & 217 & 35 & 203 \\
\hline 9 & 331 & 18 & 268 & 27 & 206 & 36 & 150 \\
\hline
\end{tabular}

The passenger volume data for the 36 traffic zones are shown in Table 1, obtained from a 2011 urban personal trip survey.

\subsection{Solution results}

The solutions for cases of $n=2,3,4$, and 5 are shown in Figure 6 and Table 2.

The profit of the coach service is the greatest with 5 trip-end routes. The profits are positively correlated with the route number, i.e., the profit increases as the number of routes increases. For example, the profit increases from 21,047 yuan in the case of $n=2$ to 41,725 yuan in the case of $n=5$.

To calculate the operating cost, it is assumed that the on-board passengers are the product of the coach capacity and the loading rate, and then, the coaches are dispatched from the stops based on the passenger flows. Therefore, the number of coaches needed can be calculated with Equation 9. However, in the

Table 2 - The trip-end network of the EC system real case, the daily minimum dispatches should be considered because a service mode must maintain a minimum service level. In other words, even if there are few passengers at a time point, the coach must still be dispatched. If the operation frequency is lower than the minimum, the service mode will be eliminated by the market completely. Thus, we should set a daily minimum dispatch of $F$ when designing the tripend route network. As a result, the daily dispatches should be the larger of the calculated criteria and the minimum criteria, which are calculated as follows.

$Q^{k}=\max \left\{\left[\frac{\sum_{i \in S_{k}} q_{b i}^{k}}{W \cdot A}\right], F\right\}, \forall k \in K$

\subsection{Sensitivity analysis}

Setting the daily minimum dispatches to be $F=6$, 8 and 10, the corresponding total profits of coach service are shown in Figure 7.

\begin{tabular}{|c|c|c|c|c|c|}
\hline & \multirow[b]{2}{*}{ Routes } & \multicolumn{4}{|c|}{ Optimization results } \\
\hline & & Stops & Passenger flows (person) & Ticket price (RMB) & $\begin{array}{l}\text { Profit of coach } \\
\text { service (RMB) }\end{array}$ \\
\hline \multirow{2}{*}{$n=2$} & Route 1 & 2-15-1-37-39 & $83-134-223$ & \multirow{2}{*}{$\begin{array}{c}91-94-105 \\
90-88-99\end{array}$} & \multirow{2}{*}{21,047} \\
\hline & Route 2 & 14-13-6-37-39 & $53-107-174$ & & \\
\hline \multirow{3}{*}{$n=3$} & Route 1 & 10- 3-11-37-39 & $62-149-210$ & 89-94-102 & \multirow{3}{*}{29,606} \\
\hline & Route 2 & 23-13-1-37-39 & $44-101-196$ & $90-88-102$ & \\
\hline & Route 3 & 20-24- 7-37-39 & 56-113-181 & $90-90-89$ & \\
\hline \multirow{4}{*}{$n=4$} & Route 1 & 4- 8-10 -37-39 & $88-154-219$ & $92-97-105$ & \multirow{4}{*}{37,755} \\
\hline & Route 2 & 14-13-11-37-39 & 58-118-192 & $90-88-97$ & \\
\hline & Route 3 & 9- 7-32 -37-39 & 66-133-178 & 89-90-93 & \\
\hline & Route 4 & 19-12- 6-37-39 & $52-115-189$ & 91-89-99 & \\
\hline \multirow{5}{*}{$n=5$} & Route 1 & 4-10-1-37-39 & $115-160-240$ & $92-95-100$ & \multirow{5}{*}{41,725} \\
\hline & Route 2 & 23-13- 6-37-39 & $47-107-182$ & $90-88-97$ & \\
\hline & Route 3 & 31- 3- 5-37-39 & $27-119-180$ & $90-88-98$ & \\
\hline & Route 4 & 22-24-11-37-39 & $48-92-156$ & $90-89-93$ & \\
\hline & Route 5 & 19-34- 7-37-39 & $52-72-135$ & $91-89-87$ & \\
\hline
\end{tabular}




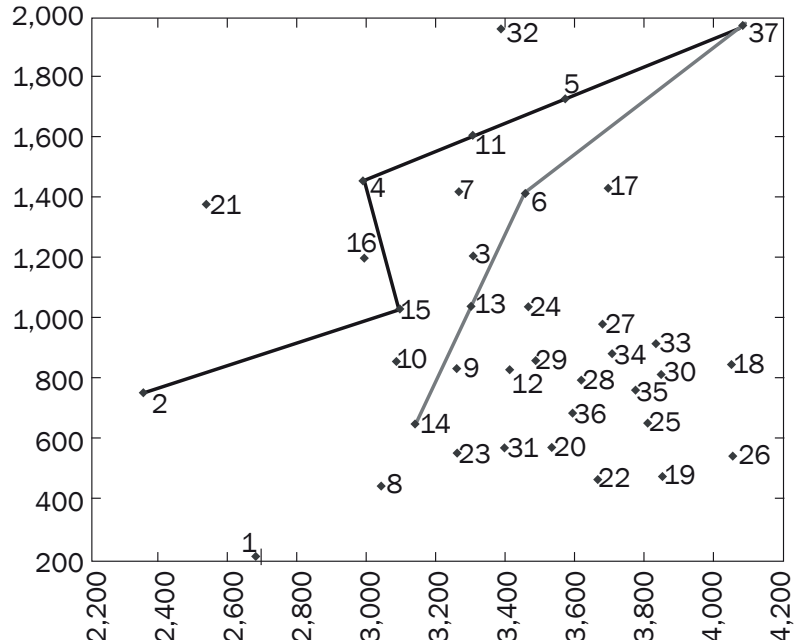

a) $n=2$

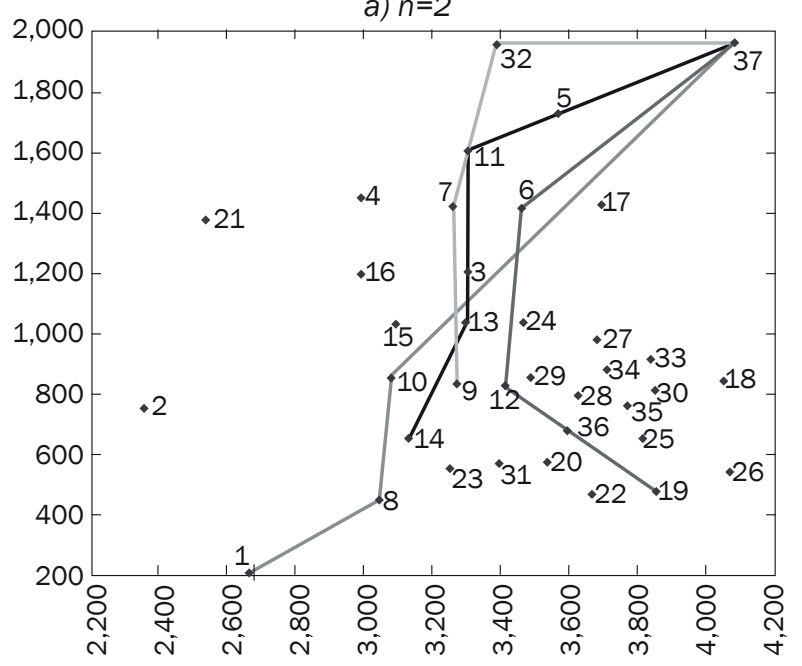

c) $n=4$

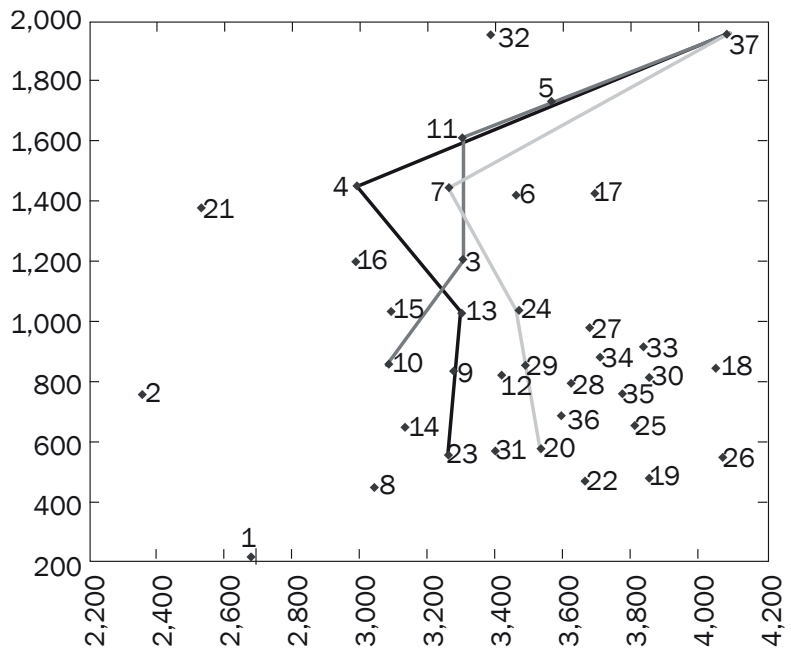

b) $n=3$

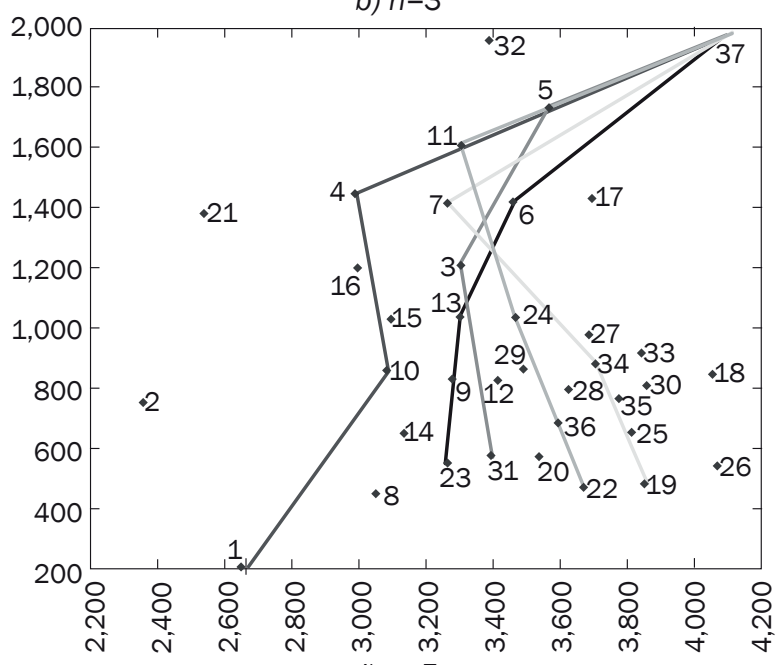

d) $n=5$

Figure 6 - Trip-end routes

The results show the following: (1) The profits initially increase and then decrease as the number of routes increases. For the three cases, the maximum profit can be obtained when $n=4$, indicating that the operation of 4 trip-end routes is the best choice regardless of how many coaches are dispatched. (2) When operating 4 trip-end routes $(n=4)$, the daily profits in the cases of $F=6,8$ and 10 are $37,755,27,955$ and 16,755 yuan, respectively, and the corresponding dispatches are 25, 32 and 40, with daily unit coach profits of 1,510, 874 and 419 yuan, respectively. The daily unit coach profit in the case of $F=6$ is the largest, indicating that although increasing the dispatches might improve the service level, it also increases the operating costs and results in decreased profit. For the case in this study,

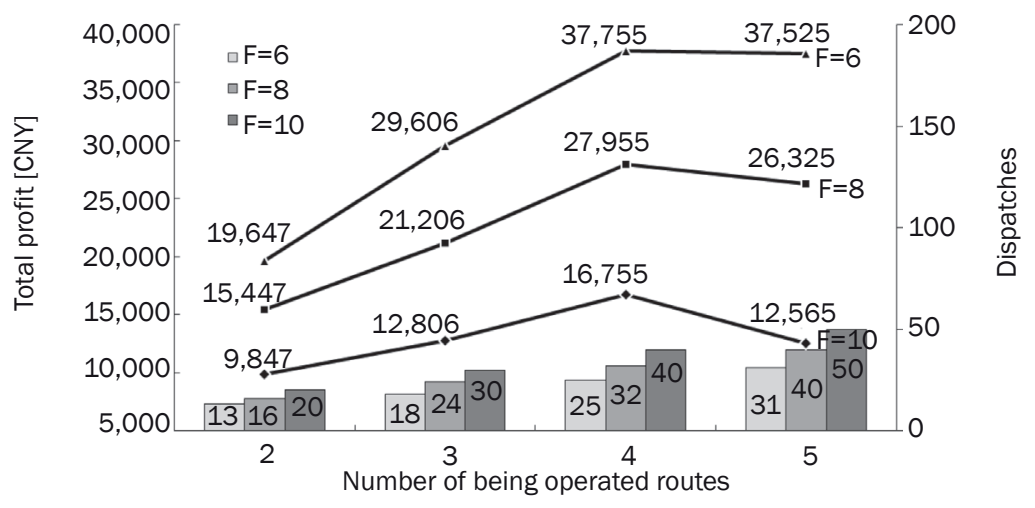

Figure 7 - Operation profits and dispatches in the cases of $F=6,8$, and 10 and $n=2-5$ 
Table 3 - Operation figures of a coach service provider in Dalian

\begin{tabular}{||l|c|c|c||}
\hline \multicolumn{1}{|c|}{ Operation Parameters } & Case 1 & Case 2 & Case 2 \\
\hline \hline Ticket price (RMB) & 115 & 99 & {$[88,105]$} \\
\hline Daily passenger volume (person) & 680 & 130 & 778 \\
\hline Daily departure coaches (coach) & 22 & 4 & 25 \\
\hline Daily operating profit (RMB) & 47,400 & 7,270 & 37,755 \\
\hline
\end{tabular}

the optimal scheme is to set 4 trip-end routes and dispatch 25 coaches $(F=6)$. In this scheme, the daily profit of the coach service is 37,755 yuan.

\subsection{Analyses of the optimization results}

Using a coach enterprise in Dalian as an example, we have calculated some business indices of its passenger transport service from Dalian to Shenyang in three situations, i.e., actual cases before and after HSR operation (Cases 1 and 2) and the case of operation with the optimized door-to-door trip-end network after HSR operation (Case 3 ). The results are shown in Table 3. The following conclusions can be drawn:

1) For ticket prices: The ticket prices for the coach in Cases 1 and 2 are 115 and 99 yuan, respectively, whereas the ticket price in Case 3 is 88-105 yuan. The designed price in Case 3 is in the rational range.

2) For passenger volume: The actual daily passengers of coach transport are 680 and 130 persons in Cases 1 and 2, respectively, whereas the calculated daily passengers are 778 in Case 3, indicating that the market share of EC will increase greatly with the door-to-door service.

3) For dispatches of coaches: There were 22 coach dispatches before the HSR operation in one business day, whereas after HSR operation, only 4 coach dispatches remained. In Case 3, 25 coach dispatches are needed, which is nearly the same as the number needed in Case 1.

4) For operation profits: The actual daily profits of EC are 47,400 and 7,270 yuan in Cases 1 and 2, respectively. However, if the EC is operated with doorto-door service after HSR operation, the daily profit will be 37,755 yuan. Thus, door-to-door service can effectively improve the profitability of ECs. Before the HSR operation, the daily unit coach profit is 2,155 yuan, whereas with the door-to-door model, it is 1,510 yuan, decreasing by 645 yuan. With door-to-door service, EC passengers can enjoy better service because they can board at stops near the origins and can enjoy more frequent dispatches with lower ticket prices.

The optimal trip-end network effectively improves EC service and thus increases the passenger volume and operating profits of the EC system. The optimized operation scheme cannot only guarantee the operation profits but also improve the EC's service level. Thus, according to the OD trips, optimizing the tripend network of the EC system to provide door-to-door service is an effective and practical method for ECs to overcome the challenges from HSR and to maintain their market shares and earn new profits.

\section{CONCLUSION}

A door-to-door operation mode and a method to optimize ECs' trip-end network under the new mode have been proposed for EC business in the HSR era. The method is a bi-level programming model with the objective of maximizing the daily profit with ECs and the travellers' trip utility. A case study has been conducted using real data from Dalian to Shenyang. In the case study, with door-to-door operation mode and the corresponding optimized route network, the market share of the EC could be increased and the EC's operating profits could be guaranteed.

Moreover, the service is also improved significantly due to the reduced ticket prices, increased dispatching frequency and easier onboard accessibility. The optimization model will provide a theoretical basis and practical method for designing the end-trip network for ECs to operate in door-to-door mode in the HSR era.

However, there are also some shortcomings in this paper. Firstly, we simplified the description of the traveller's waiting time by determining the departure frequency $\left(Q^{k}\right)$. Therefore, the waiting time is an exogenous variable. For inter-city EC it is rational to convert it to an exogenous variable by using a fixed departure frequency because, unlike bus transit, the frequency of EC does not need to be dense. In our future study, we may try to optimize the network, the ticket price and the departure frequency of the EC simultaneously and make the waiting time an endogenous variable.

Secondly, the model assumes that travellers could accurately forecast the real travel time before making their routing decision. However, in reality, the travel time is unknown to travellers. Their routing decision is based on the known EC price and travel time expectation; thus, the problem has changed into a multi-objective stochastic network optimization problem that cannot be solved by the Frank-Wolfe algorithm. We will use the biogeography-based optimization method or particle swarm optimization method $[26,27]$ to handle the stochastic network in our future study. 


\section{ACKNOWLEDGMENT}

This research is supported by the Natural Science Foundation of China (Grant No.51078049), the Fundamental Research Funds for the Central Universities (Grant No.3132016303) and Fundamental Research Funds for the Central Universities (Grant No. 3132016303). The authors want to thank the editor and anonymous reviewers for their comments.

杨忠振, 教授, 博士

邮箱: yangzz@dlmu. edu. cn

(通讯作者)

程健南, 博士

邮箱: cheng_jian_nan@dlmu. edu.cn

公路客运 “门到门”运营模式下的末端线网优化设计

摘要

高铁开通后同一交通走廊上公路客运的客流量大 幅度下降，本文提出 “门到门”的公路客运运营模 式, 并构建双层规划模型优化设计新模式下公路客 运的末端线网，最大化其日运营利润。通过对实际 案例的分析, 结果表明, 公路客运的运营利润随末 端线路数的增加先升后降。在公路客运时代, 门到 门运输可以有效的保证市场份额并且增加利润。

\section{关键词}

城际巴士; 高铁; 门到门服务; 末端线网; 票件; 营运利润

\section{REFERENCES}

[1] Lampkim S. The design of routes, undertaking: a case study. Transp Res. 1967;34(2): 123-127.

[2] Mandl CE. Evaluation and optimization of urban public transportation networks. Eur J Oper Res. 1980;5(6): 396-404.

[3] Van Nes R, Bovy PHL. The importance of objectives in urban transit network design. Transp Res Rec. 2000;1735: 50-57.

[4] Chakroborty P, Wivedi T. Optimal route network design for transit systems using genetic algorithms. Eng Optim. 2002;34(1):83-100.

[5] Ceder A, Wilson NHM. Bus network design. Transp Res B Methodol. 1986;20(4): 331-344.

[6] Baaj MH, Mahmassani HS. An Al-based approach for transit route system planning and design. J Adv Transp. 1991;25(2): 187-209.

[7] Baaj $\mathrm{MH}$, Mahmassani HS. Hybrid route generation heuristic algorithm for the design of transit networks. Transp Res C Emerg Technol. 1995;3(1): 31-50.

[8] Ceder A, Israeli Y. User and operator perspectives in transit network design. Transp Res Rec. 1998;1623: 3-7.

[9] Pattnaik SB, Mohan S, Tom VM. Urban bus transit route network design using genetic algorithm. J Transp
Eng. 1998;124(4): 368-375.

[10] Tom VM, Mohan S. Transit route network design using frequency coded genetic algo-rithm. J Transp Eng. 2003;129(2): 186-195.

[11] Bielli M, Caramia M, Carotenuto P. Genetic algorithms in bus network optimization. Transp Res C Emerg Technol. 2002;10(1): 19-34.

[12] Ngamchai S, Lovell DJ. Optimal time transfer in bus transit route network design using a genetic algorithm. J Transp Eng. 2003;129(5): 510-521.

[13] Agrawal J, Mathew TV. Transit route network design using parallel genetic algorithm. J Comput Civ Eng. 2004;18(3): 248-256.

[14] Lee YJ, Vuchic VR. Transit network design with variable demand. J Transp Eng. 2005;131(1): 1-10.

[15] dell'Olio L, Moura J, Ibeas A. Bi-level mathematical programming model for locating bus stops and optimizing frequencies. Transp Res Rec. 2006;1971(1): 23-31.

[16] Fan W, Machemehl RB. Optimal transit route network design problem with variable transit demand: genetic algorithm approach. J Transp Eng. 2006;132(1): 40-51.

[17] Fan W, Machemehl RB. Using a simulated annealing algorithm to solve the transit route network design problem. J Transp Eng. 2006;132(2): 122-132.

[18] Szeto WY, Wu Y. A simultaneous bus route design and frequency setting problem for tin Shui Wai, Hong Kong. Eur J Oper Res. 2011;209(2): 141-155.

[19] Afandizadeh S, Khaksar H, Kalantari N. Bus fleet optimization using genetic algorithm a case study of Mashhad. Int J Civ Eng. 2013;11(1): 43-52.

[20] Szeto WY, Jiang Y. Transit route and frequency design: bi-level modeling and hybrid arti-ficial bee colony algorithm approach. Transp Res B Methodol. 2014;67(9): 235-263.

[21] Arbex RO, da Cunha CB. Efficient transit network design and frequencies setting multi-objective optimization by alternating objective genetic algorithm. Transp Res B Methodol. 2015;81: 355-376.

[22] Zhang Y, Wu L, Wei G, Wang S. A novel algorithm for all pairs shortest path problem based on matrix multiplication and pulse coupled neural network. Digit Signal Process. 2011;21(4): 517-521.

[23] Ma JQ, Bai Y, Han BM. Characteristic analysis of basic unit and complex network for ur-ban rail transit. J Traffic Transp Eng. 2010;4.

[24] Dai T, Jin F. Spatial interaction and network structure evolvement of cities in terms of Chi-na's rail passenger flows. Chin Geogr Sci. 2008;18(3): 206-213.

[25] Zhao F. Large-scale transit network optimization by minimizing user cost and transfers. J Public Transp. 2006;9(2): 107-129.

[26] Wang S, Yang J, Liu G, Du S, Yan J. Multi-objective path finding in stochastic networks using a biogeography-based optimization method. Simulation. 2016;92(7): 637-647.

[27] Zhang $\mathrm{Y}$, Wu L, Wang S. UCAV path planning by fitness-scaling adaptive chaotic particle swarm optimization. Math Probl Eng. 2013;2013(2013): 705238. 